\title{
Uso de resíduos orgânicos no crescimento de mudas de Mimosa setosa
}

Júlio Cézar Tannure Faria ${ }^{1}$, Marcos Vinicius Winckler Caldeira ${ }^{1}$, William Macedo Delarmelina ${ }^{1}$, Elzimar de Oliveira Gonçalves ${ }^{1}$

${ }^{1}$ Universidade Federal do Espírito Santo, DCFM/CCA, Alto Universitário, s/nº, Guararema, Centro, CEP 29500-000, Alegre, ES, Brasil

\author{
*Autor correspondente: \\ mvwcaldeira@gmail.com \\ Termos para indexação: \\ Leguminosas \\ Produção de mudas florestais \\ Substrato para plantas \\ Sansão-de-minas \\ Index terms: \\ Leguminosas \\ Forest seedling production \\ Substrate for plants \\ Sansão-de-minas
}

\section{Histórico do artigo:}

Recebido em 15/03/2013

Aprovado em 16/12/2013

Publicado em 31/12/2013

\begin{abstract}
Resumo - O objetivo desse trabalho foi avaliar o crescimento de mudas de Mimosa setosa com uso de diferentes combinações de resíduos renováveis, sendo eles: lodo de esgoto, casca de arroz carbonizada e palha de café in natura, além do substrato comercial. As mudas foram produzidas em tubetes com capacidade de $120 \mathrm{~cm}^{3}$, permanecendo 120 dias na casa de sombra e mais 30 dias na área de rustificação. Quando as mudas atingiram 150 dias de crescimento foram avaliados: a altura da parte aérea, o diâmetro do coleto, a relação entre altura da parte aérea e diâmetro do coleto, a massa seca da parte aérea, a massa seca do sistema radicular, a massa seca total, a relação massa seca da parte aérea e massa seca do sistema radicular e o índice de qualidade de Dickson. Os dados foram submetidos à análise estatística (ANOVA) e as médias, comparadas pelo teste Scott-Knott, ao nível de 5\% de probabilidade. Os substratos formulados com lodo de esgoto, casca de arroz carbonizada e palha de café in natura propiciaram os melhores resultados das características avaliadas, sendo o tratamento com $80 \% \mathrm{LE}+10 \% \mathrm{CAC}+10 \% \mathrm{PC}$ in natura o que proporcionou as mais adequadas características morfológicas avaliadas na produção das mudas de Mimosa setosa.
\end{abstract}

\section{Use of different residues in growth of seedlings of Mimosa setosa}

\begin{abstract}
The aim of this study was to evaluate the growth of seedlings of Mimosa setosa using different combinations of residues renewable, which are: sewage sludge, carbonized rice husk and straw coffee in natura, and commercial substrate. The seedlings were grown in plastic tubes with a capacity of $120 \mathrm{~cm}^{3}$, remaining 120 days under shadow in a greenhouse and more 30 days in rustification area. When the seedlings reached 150 days of growth were measured: the shoot height, the diameter, the ratio of shoot height and diameter, the shoot dry weight, the root dry weight, the total dry mass, the ratio dry shoot mass and root dry mass and the Dickson quality index. The data were subjected to statistical analysis (ANOVA) and the means were compared by the Scott-Knott test, at $5 \%$ probability. The substrates made with sewage sludge, carbonized rice hull and coffee straw provided better results of growth of seedlings. Moreover, the treatment with $80 \%$ of sewage sludge mixed with $10 \%$ carbonized rice husk and $10 \%$ straw coffee in natura provided the best development of the morphological characteristics evaluated, and it was recommended for the production of Mimosa setosa.
\end{abstract}




\section{Introdução}

A espécie Mimosa setosa Benth, também conhecida como sansão-de-minas, é uma espécie nativa do Brasil com características desejáveis para recuperação de áreas degradadas. Localizada nas regiões norte, nordeste, centro-oeste e sudeste do Brasil, o sansão-deminas recebe esse nome devido sua ampla ocorrência no Estado de Minas Gerais (Dutra \& Morim, 2010). $\mathrm{O}$ uso de espécies leguminosas na recuperação de áreas degradadas vem sendo cada vez mais frequente devido a suas vantagens, como rápido crescimento e recobrimento do solo, capacidade de fixação de nutrientes, principalmente o nitrogênio, e auxílio contra ação de plantas invasoras (Franco et al., 1994; Nau \& Sevegnani, 1997).

A produção de mudas constitui uma das etapas mais importantes do sistema produtivo, sendo altamente dependente da utilização de insumos (Silveira et al., 2002).

A utilização de substratos renováveis, principalmente os constituídos por resíduos orgânicos, vem crescendo gradativamente nos viveiros, pois além de atenderem as reais necessidades de fertilidade e nutrição das plantas, também atendem as questões ambientais sobre os impactos causados pelo acúmulo de lixo gerado pelas atividades humanas (Ceconi et al., 2006; Krefta et al., 2012; Kratz \& Wendling, 2013).

O lodo de esgoto, após sofrer um processo de estabilização, resulta em uma parte sólida que pode ser usada como condicionador das propriedades físicas, químicas e biológicas do solo, considerando seu teor de matéria orgânica e nutrientes (Melo et al., 1994). No processo de produção de mudas de espécies florestais, o uso de lodo de esgoto tem sido uma alternativa viável como fonte de matéria orgânica e de nutrientes (Teles et al., 1999; Kratz, 2011; Trazzi, 2011; Caldeira et al., 2012a; 2012b; 2012c; Caldeira et al., 2013a; 2013b), demonstrando resultados satisfatórios na formulação de substratos (Guerrini \& Trigueiro, 2003; Caldeira et al., 2012c).

Admitindo-se a importância do substrato na produção de mudas de espécies florestais, propôs-se no presente estudo verificar o potencial do uso de resíduos orgânicos na formulação de substratos para a produção de mudas de Mimosa setosa, bem como analisar as propriedades químicas e físicas dos componentes dos substratos.

\section{Material e métodos}

O experimento foi instalado no viveiro florestal do Departamento de Ciências Florestais e da Madeira do Centro de Ciências Agrárias da Universidade Federal do Espírito Santo, localizado no Município de Alegre, ES, com altitude média de $277 \mathrm{~m}$. O clima enquadra-se no tipo Cwa (inverno seco e verão chuvoso), de acordo com a classificação de Köppen, com precipitação anual média de $1.104 \mathrm{~mm}$ e temperatura média anual de $24,1^{\circ} \mathrm{C}$, com máximas diárias de $31,0{ }^{\circ} \mathrm{C}$ e mínimas de $20,2{ }^{\circ} \mathrm{C}$ (Maia et al., 2007).

Os tratamentos foram formulados utilizando lodo de esgoto (LE), palha de café in natura (PC in natura) e casca de arroz carbonizada (CAC), além de substrato comercial MecPlant (SC) $(60 \%$ de composto de casca de pinus, $15 \%$ de vermiculita e $25 \%$ de húmus mais solo vegetal).

O lodo de esgoto utilizado foi proveniente da Estação de Tratamento de Esgoto da empresa Foz do Brasil S.A., localizada no Município de Cachoeiro de Itapemirim, região sul do Estado do Espírito Santo. O resultado da análise química do lodo de esgoto pode ser visualizado na Tabela 1 que, com base na resolução CONAMA $375 / 2006$, está apto para uso em ambientes agrícolas, exceto para culturas alimentícias. O material ficou exposto a pleno sol por 30 dias e foi posteriormente passado por uma peneira de aço com malha de $2 \mathrm{~mm}$ para homogeneização das partículas.

A casca de arroz carbonizada com granulometria entre $1 \mathrm{~mm}$ e $2 \mathrm{~mm}$ (original) e o substrato comercial foram procedentes da Fibria S.A. (Unidade Aracruz), além da palha de café in natura com granulometria de $4 \mathrm{~mm}$, adquirida na região de Muniz Freire, ES.

$\mathrm{O}$ experimento foi instalado em delineamento inteiramente casualizado, constituído de 10 tratamentos (Tabela 2), com seis repetições, cada uma com oito plantas.

No momento da mistura dos componentes, foram adicionados $750 \mathrm{~g}$ de sulfato de amônio $(20 \% \mathrm{~N}) ; 1.667 \mathrm{~g}$ de superfosfato simples $\left(18 \% \mathrm{P}_{2} \mathrm{O}_{5}\right)$ e $100 \mathrm{~g}$ de cloreto de potássio $\left(60 \% \mathrm{~K}_{2} \mathrm{O}\right)$ por $\mathrm{m}^{3}$ de substrato, conforme recomendação proposta por Gonçalves et al. (2000), para o Eucalyptus sp. Optou-se por utilizar fertilizantes granulados, pela facilidade de homogeneização no substrato. A calagem, adubação com micronutrientes e adubação de cobertura não foram realizadas. 
Tabela 1. Análise química do lodo de esgoto de filtro anaeróbico proveniente da Foz do Brasil S.A. na Estação de Tratamento de Esgoto de Cachoeiro de Itapemirim, ES.

\begin{tabular}{|c|c|c|}
\hline Elementos & Resultados analíticos ${ }^{1}$ & Resolução no 375/2006 CONAMA ${ }^{2}$ \\
\hline Arsênio & $<0,5 \mathrm{mg} \mathrm{dm}^{-3}$ & $41 \mathrm{mg} \mathrm{kg}^{-1}$ \\
\hline Bário & $156 \mathrm{mg} \mathrm{dm}^{-3}$ & $1300 \mathrm{mg} \mathrm{kg}^{-1}$ \\
\hline Cádmio & $<0,053 \mathrm{mg} \mathrm{dm}^{-3}$ & $39 \mathrm{mg} \mathrm{kg}^{-1}$ \\
\hline Chumbo & $29 \mathrm{mg} \mathrm{dm}^{-3}$ & $300 \mathrm{mg} \mathrm{kg}^{-1}$ \\
\hline Cobre & $98 \mathrm{mg} \mathrm{dm}^{-3}$ & $1500 \mathrm{mg} \mathrm{kg}^{-1}$ \\
\hline Cromo & $26 \mathrm{mg} \mathrm{dm}^{-3}$ & $1000 \mathrm{mg} \mathrm{kg}^{-1}$ \\
\hline Molibdênio & $3,5 \mathrm{mg} \mathrm{dm}^{-3}$ & $50 \mathrm{mg} \mathrm{kg}^{-1}$ \\
\hline Níquel & $11 \mathrm{mg} \mathrm{dm}^{-3}$ & $420 \mathrm{mg} \mathrm{kg}^{-1}$ \\
\hline Selênio & $<0,5 \mathrm{mg} \mathrm{dm}^{-3}$ & $100 \mathrm{mg} \mathrm{kg}^{-1}$ \\
\hline Zinco & $409 \mathrm{mg} \mathrm{dm}^{-3}$ & $2800 \mathrm{mg} \mathrm{kg}^{-1}$ \\
\hline Fósforo total & $4128 \mathrm{mg} \mathrm{dm}^{-3}$ & - \\
\hline pH (suspensão a 5\%) & 5,2 & - \\
\hline Enxofre & $1,30 \%$ & - \\
\hline Nitrogênio total Kjeldahl & $5646 \mathrm{mg} \mathrm{dm}^{-3}$ & - \\
\hline Nitrogênio amoniacal & $60 \mathrm{mg} \mathrm{dm}^{-3}$ & - \\
\hline Carbono orgânico total & $16 \%$ & - \\
\hline Potássio & $1623 \mathrm{mg} \mathrm{dm}^{-3}$ & - \\
\hline Sódio & $399 \mathrm{mg} \mathrm{dm}^{-3}$ & - \\
\hline
\end{tabular}

${ }^{1}$ Resultados com base seca fornecidos pela Foz do Brasil S.A.; ${ }^{2}$ Limites máximos de concentração exigido pelo CONAMA 375/2006 (Conselho Nacional de Meio Ambiente, 2006).

Tabela 2. Proporção dos componentes para composição dos tratamentos utilizados na produção de mudas de Mimosa setosa (Alegre, ES).

\begin{tabular}{lccc}
\hline \multirow{2}{*}{ Tratamento } & LE $^{\mathbf{1}}$ & CAC $^{2}$ & PCN $^{3}$ \\
\cline { 2 - 4 } & \multicolumn{3}{c}{ \% volumétrica } \\
\hline T1 (80\%LE+10\%CAC+10\%PC in natura) & 80 & 10 & 10 \\
T2 (60\%LE+20\%CAC+20\%PC in natura) & 60 & 20 & 20 \\
T3 (40\%LE+30\%CAC+30\%PC in natura) & 40 & 30 & 30 \\
T4 (20\%LE+40\%CAC+40\%PC in natura) & 20 & 40 & 40 \\
T5 (100\%LE) & 100 & - & - \\
T6 (80\%LE+20\%PC in natura) & 80 & - & 20 \\
T7 (60\%LE+40\%PC in natura) & 60 & - & 40 \\
T8 (40\%LE+60\%PC in natura) & 40 & - & 60 \\
T9 (20\%LE+80\%PC in natura) & 20 & - & 80 \\
T10 (Substrato comercial) & Substrato comercial (SC) \\
\hline
\end{tabular}

${ }^{1}$ Lodo de esgoto, ${ }^{2}$ casca de arroz carbonizada, ${ }^{3}$ palha de café in natura.

Para determinação dos teores totais de nutrientes presentes nos componentes foi utilizada a metodologia proposta pela Claessen (1997) (Tabela 3). As análises foram realizadas no laboratório de Recursos Hídricos do DCFM/CCA-UFES, Jerônimo Monteiro, ES, sendo que os resíduos foram submetidos às análises antes da preparação dos substratos.
A caracterização física dos resíduos foi realizada no laboratório de Substratos do Departamento de Horticultura e Silvicultura da Universidade Federal do Rio Grande do Sul (UFRGS), conforme metodologia descrita na Instrução Normativa SDA N ${ }^{0} 17$ do Ministério da Agricultura, Pecuária e Abastecimento (Brasil, 2007) (Tabela 4). 
Tabela 3. Teores totais de macro e micronutrientes, matéria orgânica $(\mathrm{MO})$ e relação $\mathrm{C} / \mathrm{N}$ dos resíduos antes da instalação do experimento (Alegre, ES).

\begin{tabular}{|c|c|c|c|c|c|c|c|c|c|c|c|c|c|}
\hline \multirow{2}{*}{ Componentes } & $\mathbf{N}$ & $\mathbf{P}$ & $\mathbf{K}$ & $\mathbf{C a}$ & Mg & $\mathbf{S}$ & $\mathbf{Z n}$ & $\mathbf{F e}$ & Mn & $\mathrm{Cu}$ & B & MO & $\mathrm{C} / \mathrm{N}$ \\
\hline & \multicolumn{6}{|c|}{$\mathrm{g} \mathrm{kg}^{-1}$} & \multicolumn{5}{|c|}{ mg kg-1 } & \multicolumn{2}{|c|}{$\mathrm{g} \mathrm{kg}^{-1}$} \\
\hline $\mathrm{SC}^{1}$ & 8,8 & 1,6 & 1,2 & 8,3 & 4,1 & 0,06 & 44 & 9200 & 199 & 10 & 14 & 145,9 & 9,6 \\
\hline $\mathrm{LE}^{2}$ & 13,3 & 2,5 & 0,8 & 8,9 & 2,4 & 0,14 & 231 & 17480 & 157 & 53 & 8 & 65,5 & 2,9 \\
\hline $\mathrm{CAC}^{3}$ & 4,6 & 1,1 & 6,5 & 2,1 & 1 & 0,06 & 44 & 506 & 492 & 8 & 6 & 320,5 & 40,4 \\
\hline $\mathrm{PC}$ in natura ${ }^{4}$ & 11,6 & 1,1 & 0,8 & 29 & 4,2 & 0,4 & 25 & 7625 & 79 & 6 & 11,1 & 300 & 15,5 \\
\hline
\end{tabular}

${ }^{1}$ Substrato comercial, ${ }^{2}$ lodo de esgoto, ${ }^{3}$ casca de arroz carbonizada, ${ }^{4}$ palha de café in natura.

Tabela 4. Valores médios de volume total de poros (VTP), macroporosidade (MAC), microporosidade (MIC) e densidade (DENS) dos componentes utilizados na formulação do substrato (Alegre, ES).

\begin{tabular}{|c|c|c|c|c|}
\hline \multirow{2}{*}{ Tratamentos } & VTP & MAC & MIC & \multirow{2}{*}{$\begin{array}{l}\text { DENS } \\
\mathrm{g} \mathrm{cm}^{-3}\end{array}$} \\
\hline & \multicolumn{3}{|c|}{$\%$} & \\
\hline $\mathrm{SC}^{1}$ & 85 & 33 & 52 & 0,32 \\
\hline $\mathrm{LE}^{2}$ & 75 & 23 & 52 & 0,21 \\
\hline $\mathrm{CAC}^{3}$ & 71 & 55 & 16 & 0,12 \\
\hline PC in natura ${ }^{4}$ & 44 & 23 & 21 & 0,02 \\
\hline
\end{tabular}

${ }^{1}$ Substrato comercial, ${ }^{2}$ lodo de esgoto, ${ }^{3}$ casca de arroz carbonizada, ${ }^{4}$ palha de café in natura.

As sementes de Mimosa setosa foram doadas pela Reserva Natural Vale e passaram por um processo de quebra de dormência em ácido sulfúrico (95-97\%) por $10 \mathrm{~min}$. Posteriormente, as sementes foram lavadas com água estéril.

A semeadura foi realizada diretamente, sendo semeadas três sementes por recipiente. Após a germinação ( \pm 20 dias) foi realizado o raleio, deixando uma muda por recipiente. Os substratos correspondentes aos tratamentos foram alocados em tubetes com capacidade para $120 \mathrm{~cm}^{3}$ de substrato, sendo acondicionados em bandejas de polipropileno com capacidade para 54 tubetes espaçados. Estas bandejas foram dispostas em canteiros suspensos a $80 \mathrm{~cm}$ do solo dentro da casa de sombra, coberta com tela que permite a passagem de $75 \%$ da luminosidade. As mudas permaneceram na casa de sombra durante 120 dias e mais 30 dias na área de rustificação, sendo irrigadas quatro vezes ao dia, por sistema de irrigação automático. Em dias chuvosos, a irrigação foi cessada.

Quando as mudas atingiram 150 dias após a semeadura, foram mensuradas as seguintes características: altura da parte aérea $(\mathrm{H})$, diâmetro do coleto (DC), relação entre altura da parte aérea e diâmetro do coleto (RHDC), massa seca da parte aérea (MSPA), massa seca do sistema radicular (MSR), massa seca total (MST), relação entre massa seca da parte aérea e massa seca do sistema radicular (RMSPAR) e índice de qualidade de Dickson (IQD).

O diâmetro do coleto foi obtido com paquímetro digital e a altura com régua, tomando-se como padrão a gema terminal (meristema apical). Para a obtenção da massa seca da parte aérea e da massa seca do sistema radicular foi realizada a pesagem das partes vegetais em separado após a secagem destas em estufa de circulação de ar forçada a $70^{\circ} \mathrm{C}$, por um período de aproximadamente $72 \mathrm{~h}$. O índice de qualidade de Dickson foi obtido pela fórmula de Dickson et al. (1960):

$$
I Q D=\frac{M S T(g)}{H(\mathrm{~cm}) / D C(\mathrm{~mm})+M S P A(g) / M S R(g)}
$$

Em que: $\operatorname{MST}(\mathrm{g})=$ massa seca total; $\mathrm{H}(\mathrm{cm})=$ altura; $\mathrm{DC}(\mathrm{mm})=$ diâmetro do coleto; $\operatorname{MSPA}(\mathrm{g})=$ massa seca da parte aérea; $\operatorname{MSR}(\mathrm{g})=$ massa seca da raiz.

Os dados foram submetidos à análise estatística (ANOVA) através da comparação de médias pelo teste Scott-Knott, em nível de 5\% de probabilidade.

\section{Resultados e discussão}

$\mathrm{Na}$ avaliação do crescimento em altura da parte aérea, as mudas de Mimosa setosa apresentaram diferenças significativas entre os substratos estudados, com valores médios entre $9,32 \mathrm{~cm}$ e $58,85 \mathrm{~cm}$. A maior média de crescimento foi obtida no tratamento $\mathrm{T} 1$ $(80 \% \mathrm{LE}+10 \% \mathrm{CAC}+10 \% \mathrm{PCN})$ sendo estatisticamente superior aos demais tratamentos do experimento. $\mathrm{O}$ tratamento $\mathrm{T} 2(60 \% \mathrm{LE}+20 \% \mathrm{CAC}+20 \% \mathrm{PC}$ in natura $)$ obteve a segunda melhor média de crescimento, também apresentando alta média nessa característica morfológica. O menor resultado foi obtido pelo tratamento controle T10 (100\%SC) (Tabela 5). 
Tabela 5. Altura (H), diâmetro do coleto (DC), relação altura/diâmetro (RHDC) e índice de qualidade de Dickson (IQD) de mudas de Mimosa setosa aos 150 dias de idade (Alegre, ES).

\begin{tabular}{lcccc}
\hline \multicolumn{1}{c}{ Tratamento } & H $(\mathbf{c m})$ & DC $(\mathbf{m m})$ & RHDC & IQD \\
\hline T1 (80\%LE+10\%CAC+10\%PC in natura $)$ & $58,85 \mathrm{a}$ & $3,15 \mathrm{a}$ & $18,85 \mathrm{a}$ & $0,63 \mathrm{c}$ \\
T2 (60\%LE+20\%CAC+20\%PC in natura $)$ & $55,72 \mathrm{~b}$ & $3,11 \mathrm{a}$ & $18,01 \mathrm{a}$ & $0,61 \mathrm{c}$ \\
T3 $(40 \% \mathrm{LE}+30 \% \mathrm{CAC}+30 \% \mathrm{PC}$ in natura $)$ & $49,92 \mathrm{c}$ & $2,97 \mathrm{a}$ & $16,86 \mathrm{~b}$ & $0,66 \mathrm{c}$ \\
T4 (20\%LE+40\%CAC+40\%PC in natura $)$ & $47,16 \mathrm{~d}$ & $3,20 \mathrm{a}$ & $15,05 \mathrm{c}$ & $0,79 \mathrm{~b}$ \\
T5 (100\%LE) & $41,77 \mathrm{e}$ & $2,88 \mathrm{a}$ & $14,77 \mathrm{c}$ & $0,73 \mathrm{~b}$ \\
T6 (80\%LE+20\%PC in natura $)$ & $41,28 \mathrm{e}$ & $2,82 \mathrm{~b}$ & $14,68 \mathrm{c}$ & $0,72 \mathrm{~b}$ \\
T7 (60\%LE+40\%PC in natura $)$ & $42,31 \mathrm{e}$ & $2,71 \mathrm{~b}$ & $15,81 \mathrm{c}$ & $0,67 \mathrm{c}$ \\
T8 (40\%LE+60\%PC in natura $)$ & $38,95 \mathrm{f}$ & $2,70 \mathrm{~b}$ & $14,51 \mathrm{c}$ & $0,75 \mathrm{~b}$ \\
T9 (20\%LE+80\%PC in natura $)$ & $40,19 \mathrm{f}$ & $2,64 \mathrm{~b}$ & $15,50 \mathrm{c}$ & $0,71 \mathrm{~b}$ \\
T10 (Substrato comercial) & $9,32 \mathrm{~g}$ & $1,35 \mathrm{c}$ & $7,26 \mathrm{~d}$ & $1,29 \mathrm{a}$ \\
\hline \multicolumn{1}{c}{ CV\% } & 7,50 & 12,73 & 13,65 & 19,71 \\
\hline
\end{tabular}

Médias seguidas da mesma letra, na coluna, não diferem estatisticamente entre si pelo teste Scott-Knott $(\mathrm{P}>0,05)$.

Resultados semelhantes aos do presente estudo foram encontrados por Caldeira et al. (2013b), avaliando substratos alternativos na produção de mudas de Chamaecrista desvauxii, onde as melhores formulações dos substratos testados foram apresentadas no tratamento com $80 \%$ lodo de esgoto, $10 \%$ casca de arroz carbonizada, $10 \%$ palha de café in natura e no tratamento com $60 \%$ lodo de esgoto, $20 \%$ casca de arroz carbonizada, $20 \%$ palha de café in natura. Gomes et al. (2013), avaliando o crescimento das características morfológicas de mudas de Tectona grandis, obtiveram os melhores resultados em altura da parte aérea no substrato formulado com $80 \%$ lodo de esgoto e $20 \%$ de casca de arroz carbonizada.

Os resultados positivos apresentados nos tratamentos T1 (in natura) e T2 (in natura) podem ser explicados pelos bons teores de matéria orgânica (MO) encontrados nos componentes palha de café in natura e casca de arroz carbonizada, associados a um adequado nível de porosidade oferecido pela casca de arroz carbonizada, considerado material leve e poroso (Gonçalves \& Poggiani, 1996; Badocha et al., 2003; Guerrini; Trigueiro, 2004; Faustino et al., 2005). Além disso, os altos níveis de $\mathrm{N}$ presente nos componentes orgânicos lodo de esgoto e palha de café in natura ajudaram a proporcionar bons crescimentos em altura das mudas de $M$. setosa (Tabela 3).

Na produção de mudas de espécies florestais é recomendado que a altura da parte aérea seja de 15 a $30 \mathrm{~cm}$ para serem levadas a campo (Gomes \& Paiva, 2004). Mudas de espécies leguminosas com o objetivo de recuperação de áreas degradadas tem por meta o recobrimento do solo em um menor intervalo de tempo, não havendo uma recomendação de qual altura e diâmetro do coleto são considerados ideais para seu plantio em campo.

Quanto à característica morfológica diâmetro do coleto, as médias de crescimento das mudas de $M$. setosa variaram de 1,35 $\mathrm{mm}$ e 3,20 $\mathrm{mm}$. Os substratos T1 a T5 foram estatisticamente superiores aos demais tratamentos analisados para essa característica (Tabela 5). Os tratamentos que utilizaram palha de café in natura com o lodo de esgoto apresentaram médias estatisticamente iguais, mas inferiores aos tratamentos T1 a T5. A menor média de crescimento foi novamente do tratamento controle T10.

De acordo com a recomendação de Daniel et al. (1997), o diâmetro de coleto é uma característica morfológica que indica a capacidade de sobrevivência das mudas no campo, devendo ser maior que $2 \mathrm{~mm}$. No presente estudo, apenas o tratamento T10 (100\% SC) apresentou resultado médio inferior a $2 \mathrm{~mm}$ de crescimento no diâmetro de coleto das mudas de M. setosa (Tabela 4), indicando qualidade inferior das mudas.

Uma das possíveis explicações para o menor crescimento das características morfológicas das mudas do $\mathrm{T} 10(100 \%$, SC), principalmente a altura da parte aérea e o diâmetro de coleto, pode estar relacionado aos baixos níveis de $\mathrm{N}$ e $\mathrm{P}$ do substrato comercial, principalmente em relação ao lodo de esgoto (Tabela 3). Esses elementos são nutrientes essenciais para as atividades metabólicas das plantas. 
Nóbrega et al. (2007) observaram uma tendência de aumento no diâmetro de coleto de mudas de aroeira (Schinus terebinthifolius) com a adição de lodo de esgoto ao solo. Trigueiro \& Guerrini (2003) verificaram maior diâmetro de coleto, trabalhando com mudas de Eucalyptus grandis, nos substratos contendo de 40 a $50 \%$ de lodo de esgoto combinado com casca de arroz carbonizada. Alguns estudos na literatura mostram que o lodo de esgoto possui efeitos positivos na produção de mudas de espécies florestais (Santos, 2013; Peroni, 2012; Caldeira et al., 2012a; Faustino et al., 2005). O mesmo ocorreu nos resultados do presente estudo com mudas de $M$. setosa, tanto para crescimento em diâmetro do coleto como para altura da parte aérea.

O lodo de esgoto apesar de apresentar atributos químicos satisfatórios (Tabela 3), necessita da mistura com outros componentes a fim de dar equilíbrio entre o fornecimento de nutrientes e condições físicas, como aeração e retenção de água (Kratz \& Wendling, 2013; Peroni, 2012; Caldeira et al. 2012b). Segundo Guerrini \& Trigueiro (2004), o aumento na proporção de lodo de esgoto proporciona elevação da densidade e do percentual de microporos e, consequentemente, da capacidade de retenção de água. Alguns resíduos orgânicos leves, de baixa densidade, como a casca de arroz carbonizada e a palha de café, aumentam a macroporosidade das misturas, reduzindo assim a capacidade de retenção de água do substrato (Gonçalves et al., 2000).

Para a relação altura da parte aérea e o diâmetro do coleto (RHDC) os valores médios das mudas de $M$. setosa variaram entre 7,26 e 18,85, estando estes valores muito acima da faixa considerada ideal por Carneiro (1995), que classifica valores de RHDC entre 5,4 e 8,1 como intervalo de balanceamento de crescimento entre a altura e o diâmetro das mudas. Os altos valores encontrados no presente estudo ocorreram provavelmente devidos aos altos níveis de $\mathrm{N}$ que tendem a promover maior crescimento em altura e menor em diâmetro de coleto (Carneiro, 1995; Marques et al., 2009), o que acarreta baixo nível de robustez da muda e, consequentemente, diminui a capacidade de sobrevivência em campo.

Na literatura ocorre uma discordância a respeito do valor ideal para a RHDC. De acordo com Birchler et al. (1998), as mudas devem apresentar uma relação com valor de 10, o qual é o padrão ideal recomendado. Por outro lado, Gomes \& Paiva (2004) afirmam que quanto menor for o RHDC, maior será a capacidade das mudas sobreviverem e se estabelecerem em campo.
Trigueiro \& Guerrini (2003) e Peroni (2012) estudando mudas de Eucalyptus grandis, também obtiveram valores de RHDC superiores a faixa recomendada por Carneiro (1995) em seus experimentos, demonstrando assim, que este índice de qualidade pode não ser o mais adequado para avaliação de algumas espécies florestais, como o caso das mudas do presente estudo de M. setosa. A menor relação foi observada nas mudas produzidas com substrato comercial (Tabela 5).

Os valores médios de IQD para mudas de M. setosa variaram entre 0,61 e 1,29. O tratamento T10 $(100 \% \mathrm{SC})$ foi o que apresentou o maior valor para este índice. Quanto maior o valor de IQD, melhor será a qualidade da muda e, segundo Fonseca et al. (2002), esse parâmetro é um bom indicador de qualidade por considerar a robustez e o equilíbrio da distribuição da biomassa na muda, ponderando os resultados de várias características morfológicas importantes empregadas para avaliação da qualidade (Hunt 1990; Aguiar et al., 2011).

Estudando qualidade de mudas das espécies Pseudotsuga menziessi e Picea abies, Gomes \& Paiva (2004) avaliaram algumas características morfológicas e salientaram que o IQD deve apresentar o valor mínimo de 0,20. Desta forma, todos os tratamentos analisados no presente estudo estão dentro do ideal proposto por esses autores (Tabela 5).

O IQD é uma característica que varia em função da espécie, do manejo das mudas no viveiro, do tipo e proporção do substrato, do volume do recipiente e, principalmente, com a idade em que muda foi avaliada (Kratz \& Wendling, 2013; Caldeira et al., 2012b; Trazzi et al., 2012, Caldeira et al., 2008, Saidelles et al., 2009). Neste sentido, não é possível afirmar que o tratamento T10 apresentou a maior qualidade das mudas que os demais tratamentos, principalmente por este ter apresentado as menores médias em outras características morfológicas avaliadas no estudo.

A massa seca da parte área (MSPA) das mudas de $M$. setosa apresentaram resultados médios entre 5,221 a $6,459 \mathrm{~g}$. O tratamento $\mathrm{T} 1$ equiparou-se estatisticamente ao tratamento $\mathrm{T} 4$, sendo ambos os que apresentaram os maiores resultados. $\mathrm{O}$ tratamento que apresentou a menor média de MSPA foi o tratamento T10 (Tabela 6).

Resultados semelhantes ao presente estudo são encontrados por Caldeira et al. (2013b), avaliando substratos alternativos na produção de mudas de Chamaecrista desvauxii, onde os melhores resultados foram apresentados no tratamento composto com $60 \%$ de 
lodo de esgoto, $20 \%$ de casca de arroz carbonizada e $20 \%$ palha de café in natura. Gomes et al. (2013), testando diferentes substratos na produção de mudas de Tectona grandis, obtiveram melhores crescimentos médios em MSPA nos tratamentos em que foi utilizada casca de arroz carbonizada na composição dos substratos, tendo destaque a formulação do tratamento com $80 \%$ de lodo de esgoto e $20 \%$ de casca de arroz carbonizada e do tratamento com $60 \%$ de lodo de esgoto e $40 \%$ de casca de arroz carbonizada.

Tabela 6. Massa seca parte aérea (MSPA), massa seca radicular (MSR), massa seca total (MST), relação massa seca da parte aérea/raiz (RMSPAR) e relação massa seca da raiz/parte aérea (RMSRPA) de mudas de Mimosa setosa aos 150 dias de idade (Alegre, ES).

\begin{tabular}{|c|c|c|c|c|}
\hline Tratamento & $\operatorname{MSPA}(g)$ & MSR (g) & MST (g) & RMSPAR \\
\hline $\mathrm{T} 1(80 \% \mathrm{LE}+10 \% \mathrm{CAC}+10 \% \mathrm{PC}$ in natura $)$ & $6,459 \mathrm{a}$ & $6,110 \mathrm{a}$ & $12,569 \mathrm{a}$ & $1,06 \mathrm{c}$ \\
\hline $\mathrm{T} 2(60 \% \mathrm{LE}+20 \% \mathrm{CAC}+20 \% \mathrm{PC}$ in natura $)$ & $6,087 \mathrm{~b}$ & $5,557 \mathrm{c}$ & $11,645 \mathrm{~b}$ & $1,08 \mathrm{~b}$ \\
\hline $\mathrm{T} 3(40 \% \mathrm{LE}+30 \% \mathrm{CAC}+30 \% \mathrm{PC}$ in natura $)$ & $6,067 \mathrm{~b}$ & $5,793 \mathrm{~b}$ & $11,860 \mathrm{~b}$ & $1,05 \mathrm{c}$ \\
\hline $\mathrm{T} 4(20 \% \mathrm{LE}+40 \% \mathrm{CAC}+40 \% \mathrm{PC}$ in natura $)$ & $6,390 \mathrm{a}$ & $6,059 \mathrm{a}$ & $12,450 \mathrm{a}$ & $1,05 \mathrm{c}$ \\
\hline T5 (100\%LE) & $6,016 \mathrm{~b}$ & $5,406 \mathrm{c}$ & $11,423 \mathrm{c}$ & $1,11 \mathrm{~b}$ \\
\hline $\mathrm{T} 6(80 \% \mathrm{LE}+20 \% \mathrm{PC}$ in natura $)$ & $6,156 b$ & $5,278 \mathrm{~d}$ & $11,434 \mathrm{c}$ & $1,17 \mathrm{a}$ \\
\hline $\mathrm{T} 7(60 \% \mathrm{LE}+40 \% \mathrm{PC}$ in natura $)$ & $5,969 \mathrm{~b}$ & $5,338 \mathrm{~d}$ & $11,307 \mathrm{~b}$ & $1,12 \mathrm{~b}$ \\
\hline $\mathrm{T} 8(40 \% \mathrm{LE}+60 \% \mathrm{PC}$ in natura $)$ & $6,068 \mathrm{~b}$ & $5,633 \mathrm{c}$ & $11,702 \mathrm{~b}$ & $1,08 \mathrm{c}$ \\
\hline T9 $(20 \% \mathrm{LE}+80 \% \mathrm{PC}$ in natura $)$ & $5,960 \mathrm{~b}$ & $5,564 \mathrm{c}$ & $11,524 \mathrm{c}$ & $1,07 \mathrm{c}$ \\
\hline T10 (Substrato comercial) & $5,221 \mathrm{c}$ & $4,989 \mathrm{e}$ & $10,210 \mathrm{e}$ & $1,05 \mathrm{c}$ \\
\hline $\mathrm{CV} \%$ & 5,07 & 5,19 & 4,31 & 5,41 \\
\hline
\end{tabular}

Médias seguidas da mesma letra, na coluna, não diferem estatisticamente entre si pelo teste Scott-Knott $(\mathrm{P}>0,05)$.

A massa seca radicular das mudas de M. setosa variou entre 4,989 g e 6,110 g. Os melhores tratamentos foram obtidos por T1 e T4, os quais apresentaram os melhores incrementos em massa seca radicular e massa seca da parte aérea no presente estudo. O tratamento T3 também apresentou alta média em MSR, sendo estatisticamente inferior a apenas os tratamentos T1 e T4. Os resultados desses tratamentos comprovam que a correta formulação do substrato exerce grande influência na formação e arquitetura do sistema radicular, além de melhorar à porosidade do substrato para a aeração e aderência das raízes (Hoffmann et al., 2001).

De acordo com os dados visualizados na tabela 4, a casca de arroz carbonizada apresenta maior volume de porosidade total que a palha de café in natura. Segundo Buckeridge et al. (2004), raízes primárias e raízes jovens respiram muito intensamente e para essas raízes, o oxigênio necessário para o processo respiratório, advém do próprio solo (substrato). Esses reflexos ficaram comprovados pelos melhores resultados apresentados pelos tratamentos T1, T4 e T3.

Avaliando a massa seca total das mudas do presente estudo, os tratamentos T1 e T4 apresentaram os melhores valores médios para mudas de $M$. setosa, e o tratamento T10 a menor média.

Assim como para outras características morfológicas, as propriedades físicas da casca de arroz carbonizada, principalmente da aeração/porosidade, proporcionaram aos tratamentos T1 e T4 os melhores crescimentos em MSPA e MSR para mudas de M. setosa, assim como para MST. Segundo recomendação de Gonçalves et al. (2000), o qual estabelece uma escala de valores para a interpretação das características físicas dos substratos, a casca de arroz carbonizada utilizada no presente estudo possui níveis médios de porosidade total (55\% a 75\%) e níveis altos de macroporosidade (> 40\%) (Tabela 4).

No que se refere à relação massa seca da parte aérea/massa seca radicular (RMSPAR), o tratamento T6 apresentou a maior média estatística. Os menores resultados foram obtidos nos tratamentos $\mathrm{T} 1, \mathrm{~T} 3, \mathrm{~T} 4, \mathrm{~T} 8$, T9 e T10. Segundo Tedesco et al. (1999) e Caldeira et al. (2000), a RMSPAR é comumente menor em ambiente de baixa fertilidade, podendo ser considerada estratégia da planta para retirar o máximo de nutrientes naquela condição. No presente estudo essa argumentação é valida para as mudas de M. setosa. 
Alguns autores defendem que a RMSPAR deve ser de 2 para demonstrar bom equilíbrio de crescimento entre a parte aérea e a raiz. Com base nessa afirmação, em geral, todos os tratamentos apresentaram deficiência nesse índice de qualidade, estando os mesmos com relação próxima de 1 (Tabela 6), o que indica um desequilíbrio no ganho das massas aérea e radicular (Brissete, 1984; Daniel et al., 1997; Caldeira et al., 2008).

\section{Conclusões}

Os substratos formulados com lodo de esgoto, casca de arroz carbonizada e palha de café in natura proporcionaram os maiores crescimentos das características morfológicas para as mudas de $M$. setosa.

Para a produção de mudas de Mimosa setosa recomenda-se o uso do substrato constituído de $80 \%$ de lodo de esgoto, com 10\% casca de arroz carbonizada e $10 \%$ de palha de café in natura, sendo esse o tratamento que proporcionou o melhor crescimento das características morfológicas avaliadas: altura da parte aérea, diâmetro do coleto, relação altura da parte aérea e diâmetro do coleto, massa seca da parte aérea, massa seca do sistema radicular e massa seca total.

\section{Referências}

AGUIAR, F. F. A.; KANASHIRO, S.; TAVARES, A. R.; NASCIMENTO, T, D, R.; ROCCO, F. M. Crescimento de mudas de pau-brasil (Caesalpinia echinata Lam.), submetidas a cinco níveis de sombreamento. Revista Ceres, Viçosa, MG, v. 58, n. 6, p. 729-734, 2011.

BADOCHA, T. E.; COSTA, R. S. C.; LEONIDAS, F. C. Casca de café: um importante insumo para a agricultura orgânica. In: SIMPÓSIO DE PESQUISA DOS CAFÉS DO BRASIL, 3., Porto Seguro, BA. Anais... Brasília, DF: Embrapa Café, 2003.

BIRCHLER, T.; ROSE, R. W.; ROYO, A.; PARDOS, M. La planta ideal: revisiondel concepto, parâmetros definitorios e implementaction practica. Investigacion Agraria, Sistemas y Recursos Forestales, Madrid, v. 7, n. 1/2, p. 109-121, 1998.

BRASIL. Ministério da Agricultura, Pecuária e Abastecimento. Instrução Normativa SDA No 17. Diário Oficial [da] República Federativa do Brasil, Brasília, DF, seção 1, n. 99, 24 maio 2007.

BRISSETE, J. C. Summary of discussion of about seedling quality. In: SOUTHERN NURSERY CONFERENCES, 1984, Alexandria. Proceedings... New Orleans: USDA, Forest Service/Southern Forest Experiment Station, 1984. p.127-128.

BUCKERIDGE, M. S.; TINÉ, M. A. S.; MINHOTO, M. J.; LIMA, D. U. Respiração. In: KERBAUY, G. B. (Ed.). Fisiologia vegetal. Rio de Janeiro: Guanabara Koogan, 2004. p.198-216.
CALDEIRA, M. V. W; DELARMELINA, W. M; PERONI, L.; GONÇALVES, E. O.; SILVA, A. G. Lodo de esgoto e vermiculita na produção de mudas de eucalipto. Pesquisa Agropecuária Tropical, Goiânia, v. 43, n. 2, p. 155-163, 2013 a.

CALDEIRA, M. V. W.; DELARMELINA, W. M.; FARIA, J. C. T.; JUVANHOL, R. S. Substratos alternativos na produção de mudas de Chamaecrista desvauxii. Revista Árvore, Viçosa, MG, v. 37 , n.1, p. 31-39, 2013b.

CALDEIRA, M. V. W.; DELARMELINA, W. M.; LUBE, S. G.; GOMES, D. R.; GONÇALVES, E. O; ALVES, A. F. Biossólido na composição de substrato para a produção de mudas de Tectona grandis. Floresta, Curitiba, v. 42, n. 1, p. 77-84, 2012a.

CAldeIRA, M. V. W.; PERONI, L.; GOMES, D. R.; DELARMELINA, W. M.; TRAZZI, P.A. Diferentes proporções de biossólido na composição de substratos para a produção de mudas de timbó (Ateleia glazioveana Baill). Scientia Forestalis, Piracicaba, v. 40, n. 93 , p. $15-22,2012$ b.

CALDEIRA, M. V. W.; GOMES, D. R.; GONÇALVES, E. O.; DELARMELINA, W. M.; SPERANDIO, H. V.; TRAZZI, P. A. Biossólido como substrato para produção de mudas de Toona ciliata var. australis. Revista Árvore, Viçosa, MG, v. 36, n. 6, p. 1009-1017, 2012c.

CALDEIRA, M. V. W.; ROSA, G. N.; FENILLI, T. A. B.; HARBS, R. M. P. Composto orgânico na produção de mudas de aroeira-vermelha. Scientia Agraria, Curitiba, v. 9, n. 1, p. 27-33, 2008.

CALDEIRA, M. V. W.; SCHUMACHER, M. V.; BARICHELLO, L. R.; VOGET, H. L. M.; OLIVEIRA, L. S. Crescimento de mudas de Eucalyptus saligna Smith em função de diferentes doses de vermicomposto. Floresta, Curitiba, v. 28, n. 1/2, p. 19-30, 2000.

CARNEIRO, J. G. A. Produção e controle de qualidade de mudas florestais. Curitiba: UFPR/FUPEF, 1995. p. 41-65.

CECONI, D. E.; POletTO, I.; BRUN, E. J.; LOVATO, T. Crescimento de mudas de açoita-cavalo (Luehea divaricata Mart.) sob influência da adubação fosfatada. Cerne, Lavras, v. 12, n. 3, p. 292-299, 2006

CLAESSEN, M. E. C. (Org.). Manual de métodos de análises de solo. 2. ed. Rio de Janeiro: EMBRAPA-CNPS, 1997. 212 p. (EMBRAPA-CNPS. Documentos, 1).

CONSELHO NACIONAL DE MEIO AMBIENTE. Resolução CONAMA n ${ }^{\circ} 375 / 2006$, de 30 de agosto de 2006. Define critérios e procedimentos, para o uso agrícola de lodos de esgoto gerados em estações de tratamento de esgoto sanitário e seus produtos derivados, e dá outras providências. Disponível em: < http://www.mma.gov.br/ port/conama/legislacao/CONAMA_RES_CONS_2006_375.pdf $>$. Acesso em: 20 mar. 2013.

DANIEL, O.; VITORINO, A. C. T.; ALOISI, A. A.; MAZZOCHIN, L.; TOKURA, A. M.; PINHEIRO, E. R.; SOUZA, E. F. Aplicação de fósforo em mudas de Acacia mangium. Revista Árvore, Viçosa, MG, v. 21, n. 2, p. 163-168, 1997. 
DICKSON, A.; LEAF, A. L.; HOSNER, J. F. Quality appraisal of while spruce and white pine seedling stock in nurseries. Forestry Chronicle, Canada, v. 36, p. 11-13, 1960.

DUTRA, V. F.; MORIM, M. P. Mimosa in lista de espécies da flora do Brasil. Rio de Janeiro: Jardim Botânico do Rio de Janeiro, 2010.

FAUSTINO, R.; KATO, M. T.; FLORÊNCIO, L.; GAVAZZA, S. Biossólido como substrato na produção de Senna siamea Lam. Revista Brasileira de Engenharia Agrícola e Ambiental, Campina Grande, v. 9, p. 278-282, 2005.

FONSECA, E. P.; VALÉRI, S. V.; MIGLIORANZA, E.; FONSECA, N. A. N.; COUTO, L. Padrão de qualidade de mudas de Trema micrantha (L.) Blume, produzidas sob diferentes períodos de sombreamento. Revista Árvore, Viçosa, MG, v. 26, n. 4, p. 515 523, 2002.

FRANCO, A. A.; CAMPELLO, E. F.; DIAS, L. E.; FARIA, S. M. de. Revegetação de áreas de mineração em Porto Trombetas - PA com leguminosas arbóreas noduladas e micorrizadas. In: Simpósio sul-americano e II simpósio nacional sobre recuperação de áreas degradadas, 1., 1994, Foz do Iguaçu, PR. Anais... Curitiba: FUPEF, 1994. p. $145-153$.

GOMES, D. R.; CALDEIRA, M. V. W.; GONCALVES, E. O.; DELARMELINA, W. M.; TRAZZI, P. A. Lodo de esgoto como substrato para produção de mudas de Tectona grandis L. Cerne, Lavras, v. 19, n. 1, p. 123-131, 2013.

GOMES, J. M.; PAIVA, H. N. Viveiros florestais (propagação sexuada). 2. ed. Viçosa, MG: Ed da UFV, 2004.

GONÇALVES, J. L. M.; POGGIANI, F. Substratos para produção de mudas florestais. In: SUELO CONGRESSO LATINOAMERICANO DE CIÊNCIA DO SOLO, 13., 1996, Águas de Lindóia. Resumos expandidos... Águas de Lindóia: SLCS/SBCS, 1996.

GONÇALVES, J. L. M.; SANTARELLI, E. G.; MORAES NETO, S. P.; MANARA, M. P. Produção de mudas de espécies nativas: substrato, nutrição, sombreamento e fertilização. In: GONÇALVES, J. L. M.; BENEDETTI, V. (Ed.). Nutrição e fertilização florestal. Piracicaba: IPEF, 2000. p. 309-350.

GUERRINI, I. A.; TRIGUEIRO, R. M. Uso de biossólido como substrato para produção de mudas de eucalipto. Scientia Forestalis, Piracicaba, v. 64, n. 64, p. 150-162, 2003.

GUERRINI, I. A.; TRIGUEIRO, R. M. Atributos físicos e químicos de substratos compostos por biossólidos e casca de arroz carbonizada. Revista Brasileira de Ciência do Solo, Campinas, v. 28, n. 6, p. 1069-1076, 2004.

HOFFMANN, A.; PASQUAL, M.; CHALFUN, N. N.; VIEIRA, S. S. N. Substratos na indução e desenvolvimento in vitro de raízes em dois porta-enxertos de macieira. Pesquisa Agropecuária Brasileira, Brasília, DF, v. 36, n. 11, p. 1371-1379, 2001.
HUNT, G. A. Effect of styroblock design and cooper treatment on morphology of conifer seedlings. In: TARGET SEEDLING SYMPOSIUM, MEETING OF THE WESTERN FOREST NURSERY ASSOCIATIONS, GENERAL TECHNICAL REPORT RM-200, 1990. Roseburg: Proceedings... Fort Collins: United States Departament of Agriculture, Forest Service, 1990. p. 218-222.

KRATZ, D.; WENDLING, I. Produção de mudas de Eucalyptus dunnii em substratos renováveis. Floresta, Curitiba, v. 43, n. 1, p. 125 - 136, jan./mar. 2013.

KRATZ, D. Substratos renováveis na produção de mudas de Eucalyptus benthamii Maiden et Cambage e Mimosa scabrella Benth. 2011. 121 f. Dissertação (Mestrado em Ciências Florestais) - Universidade Federal do Paraná, Curitiba.

KREFTA, S. M.; BRUN, E. J.; FACCHI, S. P. Substratos orgânicos para produção de mudas de Juqueri (Senegalia polyphylla (DC) Britton \& Rose). In: SEMINÁRIO DE INICIAÇÃO CIENTÍFICAE TECNOLÓGICADA UTFPR, 17., 2012, Curitiba. Anais... Curitiba: UTFPR, 2012.

MAIA, A. R.; LOPES, J. C.; TEIXEIRA, C. O. Efeito do envelhecimento acelerado na avaliação da qualidade fisiológica de sementes de trigo. Revista Ciência e Agrotecnologia, Lavras, v. 31 , n. 3, p. 678-684, 2007.

MARQUES, L. S.; PAIVA, H. N. de; NEVES, J. C. L.; GOMES, J. M.; SOUZA, J. H. de. Crescimento de mudas de jacaré (Piptadenia gonoacantha J.F. Macbr.) em diferentes tipos de solos e fontes e doses de nitrogênio. Revista Árvore, Viçosa, MG, v. 33, n. 1, p. 81-92, 2009.

MELO. W, J.; MARQUES, M. O.; SANTIAGO, G.; CHELI, R. A.; LEITE, S. A. S. Efeito de doses crescentes de lodo de esgoto sobre frações da matéria orgânica e CTC de um Latossolo cultivado com cana-de-açúcar. Revista Brasileira de Ciência do Solo, Campinas, v. 18, p. 449-455, 1994.

NAU, S. R.; SEVEGNANI, L. Vegetação recolonizadora em mina de argila e propostas para recuperação ambiental. In: SIMPÓSIO NACIONAL SOBRE RECUPERAÇÃO DE ÁREAS DEGRADADAS, 3., 1997, Ouro Preto. Anais... Ouro Preto: SOBRADE-SIF, 1997. p. 54-66.

NÓBREGA, R. S.A.; BOAS, R. C. V.; NÓBREGA, J. C. A.; PAULA, A. M. de.; MOREIRA, F. M. de S. Utilização de biossólido no crescimento inicial de mudas de aroeira (Schinus terebynthifolius/ Raddi). Revista Árvore, Viçosa, MG, v. 31, n. 2, p. 239-246, 2007.

PERONI, L. Substratos renováveis na produção de mudas de Eucalyptus grandis. 2012. f. Dissertação (Mestrado em Ciências Florestais) - Universidade Federal do Espírito Santo, Vitória.

SAIDELLES, F. L. F.; CALDEIRA, M. V. W.; SCHIRMER, W. N.; SPERANDIO, H. V. Casca de arroz carbonizada como substrato para produção de mudas de tamboril-da-mata e garapeira. Semina: Ciências Agrárias, v. 30, supl., p.173-1186, 2009. 
SANTOS, F. E. V. Produção de mudas de Aegiphila sellowiana Cham em diferentes substratos com lodo de esgoto. 2013. f. Dissertação (Mestrado em Ciências Florestais) - Universidade Federal do Espírito Santo, Vitória.

SILVEIRA, E. B.; RODRIGUES, V. J. L. B.; GOMES, A. M. A.; MARIANO, R. L. R.; MESQUITA, J. C. P. Pó de coco como substrato para produção de mudas de tomateiro. Horticultura Brasileira, Brasília, DF, v. 20, n. 2, p. 211-216, 2002.

TEDESCO, N.; CALDEIRA, M. V. W.; SCHUMACHER, M. V. Influência do vermicomposto na produção de mudas de caroba (Jacaranda micrantha Chamisso). Revista Árvore, Viçosa, MG, v. 23, n. 1, p. 1-8, 1999.

TELES, C. R.; COSTA, A. N.; GONCALVES, R. F. Produção de lodo de esgoto em lagoas de estabilização e o seu uso no cultivo de espécies florestais na região sudoeste do Brasil. Sanare, Curitiba, v. 12 , p. 53-60, 1999.
TRAZZI, P. A.; CALDEIRA, M. V. W.; COLOMBI, R.; GONÇALVES, E. O. Qualidade de mudas de Murraya paniculata produzidas em diferentes substratos. Floresta, Curitiba, v. 42, n. 3 , p. 621-630, 2012.

TRAZZI, P. A. Substratos renováveis na produção de mudas de Tectona grandis Linn F. Jerônimo Monteiro: UFES, 2011. 84 f. Dissertação (Mestrado em Ciências Florestais) - Universidade Federal do Espírito Santo, Jerônimo Monteiro.

TRIGUEIRO, R. de M.; GUERRINI, I. A. Uso de biossólido como substrato para produção de mudas de eucalipto. Scientia Forestalis, Piracicaba, n. 64, p. 150-162, 2003. 